\title{
Muon Capture With Improved Chiral Forces
}

\author{
Roman Skibiński*', Jacek Golak, Kacper Topolnicki, Henryk Witała \\ M. Smoluchowski Institute of Physics, Jagiellonian University, PL-30059 Kraków, Poland \\ E-mail: roman.skibinski@uj.edu.pl
}

\begin{abstract}
Muon capture on the ${ }^{2} \mathrm{H},{ }^{3} \mathrm{H}$ and ${ }^{3} \mathrm{He}$ nuclei is investigated using the state-of-the-art nucleonnucleon chiral potential with a semi-local regularization up to the fifth order of chiral expansion and with the semi-phenomenological AV18 and Urbana IX forces. Despite neglecting, in the case of the chiral force, the three-nucleon forces and using a simple model of the weak current, the obtained results show a small cut-off dependence, which is an important and desirable feature of this recently developed potential. This observation is in agreement with results worked out from the strong and electromagnetic sectors. Values of the capture rates obtained with the chiral interaction are in good agreement with ones obtained with the AV18 force and the same model of weak current.
\end{abstract}

The 26th International Nuclear Physics Conference

11-16 September, 2016

Adelaide, Australia

* Speaker.

$\dagger$ We would like to thank prof.E.Epelbaum and prof.L.Marcucci for discussions. This work is a part of the LENPIC project. It was supported from the resources of the National Science Center (Poland) under grants DEC2013/10/M/ST2/00420 and DEC-2013/11/N/ST2/03733. The numerical calculations were done on the computers of the JSC in Jülich, Germany. 


\section{Introduction}

Nearly 60 years ago L.D.Faddeev published his seminal work [1] on quantum three-body scattering in which he proposed a set of coupled integral equations known now after his name. It took many years of efforts of nuclear physicist to establish, at least numerically, reliable methods to solve Faddeev equations. It is impossible to mention here all persons and groups who gave their contribution to such investigations. Their hard work, deep insight of underlying physics and experience have built a solid foundation for our current understanding of the three-nucleon systems. The interested reader can find the extended history of these works in the Introduction of Ref [2]. Nowadays the three-nucleon bound and scattering states can be calculated for various models of two- and three-body interactions, including advanced semi-phenomenological and chiral forces. These states have been used to study strong processes like elastic nucleon-deuteron scattering and the deuteron break-up reactions $[3,4,5,2,6,7]$.

The interaction of three-nucleon systems with external probes also raised interest in the nuclear physics community [8]. The electromagnetic current operator was identified as a crucial component of the Faddeev-like equations describing electromagnetic processes involving interaction between three nucleons and electrons or photons. This, in turn, introduced new questions to theoretical calculations of the structure and importance of many-body contributions to the nuclear current. Due to the complex structure of the electromagnetic current, simplifications are usually imposed on it which weakens the predictive power of such approaches. The modern chiral approach to low-energy nuclear physics gives hope to overcome this obstacle and it seems that for the first time we have at our disposal a theoretical model within which it is possible to derive consistent forces and currents. Weak processes also captured the attention of the few-body community and have been studied actively both theoretically and experimentally [9]. Similarly to electromagnetic processes, in the case of weak reactions the nuclear current also has to be included in the theoretical analysis. Again, due to its complexity only the single nuclear current or few dominant contributions to many-body currents are usually used, see e.g. [10] for one of such approaches. However, recently substantial progress has been done by H.Krebs and collaborators, who derived the axial nuclear current emerging from the chiral effective field theory up to the forth order of chiral expansion [11].

Our group, together with collaborators, has been involved in investigations of three-nucleon systems for a quite long time. The derived formalism and numerical methods of solving Faddeev equations in momentum space allow us to research not only strong processes but also electromagnetic and weak ones. A review of some of our results can be found in $[2,12,13]$. Recently, we focused again on the weak muon-capture processes, extending our earlier works on these [14] and other reactions [15]. Currently we not only include the three-nucleon interaction when studying muon captures but also consider break-up final states for muonic ${ }^{3} \mathrm{H}$ and ${ }^{3} \mathrm{He}$ atoms decays $[16,17,18]$. In this contribution we collect, in Section 3 , results on muon capture on the ${ }^{2} \mathrm{H},{ }^{3} \mathrm{He}$ and ${ }^{3} \mathrm{He}$ nuclei, published in our recent works [16, 17, 18]. Beside other nuclear potentials we use the chiral nucleon-nucleon interaction with the semi-local regularization at the fifth order of chiral expansion $\left(\mathrm{N}^{4} \mathrm{LO}\right)$, which is the most advanced chiral force derived recently by the Bochum/Bonn group [19, 20]. This new version of the chiral interaction benefits from the improved method of regularization of its long-range part. As was shown in [20, 21, 18], this leads to an extremely small dependence of nucleon-nucleon phase shifts and observables for the elastic 
nucleon-deuteron scattering, the radiative nucleon-deuteron capture and ${ }^{3} \mathrm{He}$ photodisintegration on the regulator parameter.

\section{Formalism}

We aim to describe the capture rates for $\mu^{-}+{ }^{2} \mathrm{H} \rightarrow \mathrm{n}+\mathrm{n}+v_{\mu}, \mu^{-}+{ }^{3} \mathrm{He} \rightarrow{ }^{3} \mathrm{H}+v_{\mu}, \mu^{-}+{ }^{3}$ $\mathrm{He} \rightarrow \mathrm{n}+\mathrm{d}+v_{\mu}, \mu^{-}+{ }^{3} \mathrm{He} \rightarrow \mathrm{n}+\mathrm{n}+\mathrm{p}+v_{\mu}$ and $\mu^{-}+{ }^{3} \mathrm{H} \rightarrow \mathrm{n}+\mathrm{n}+\mathrm{n}+v_{\mu}$ processes. This can be achieved by using the Schrödinger (for the muonic ${ }^{2} \mathrm{H}$ atom) or the Faddeev (in the case of three nucleons) equations. Our formalism is described in detail in [13] and [16], so here we only briefly remind the reader of the main steps.

The nuclear matrix element for the weak current operator $j_{w}^{\lambda}$ between the initial $\left|\Psi_{i}\right\rangle$ and the final $\left|\Psi_{f}\right\rangle$ states

$$
N^{\lambda} \equiv\left\langle\Psi_{f}\left|j_{w}^{\lambda}\right| \Psi_{i}\right\rangle
$$

is the basic object in the theoretical analysis for all reactions considered here. Its knowledge, combined with the well-known leptonic part of the transition amplitude, allows one to obtain any observables for the investigated processes (see [16] for detailed expressions).

Depending on the reaction, the initial nuclear state is taken as the deuteron or the three-nucleon bound state. The deuteron wave function is a solution of the Schrödinger equation and the threenucleon bound state is given as $\left|\Psi_{i}\right\rangle=(1+P)\left|\psi_{i}\right\rangle$, where the Faddeev-component $\left|\psi_{i}\right\rangle$ fulfills (under neglecting three-nucleon force) [22] the Faddeev equation

$$
\left|\psi_{i}\right\rangle=G_{0} t P\left|\psi_{i}\right\rangle \text {. }
$$

Here $G_{0}$ is the free three-nucleon propagator, $P$ is the permutation operator and $t$-operator fulfills the Lippmann-Schwinger equation

$$
t=V+V G_{0} t
$$

with a two-nucleon potential $V$.

The neutron-neutron final scattering state for deuteron breakup is given by

$$
\left\langle\Psi_{f}\right|=\left\langle\vec{p} \vec{P}_{f}=-\vec{p}_{v} m_{1} m_{2}\right|\left(1+t_{n n} G_{0}^{n n}\right)
$$

with $G_{0}^{n n}$ and $t_{n n}$ being the two-neutron free propagator and the two-neutron $t$-operator, respectively. Further, $m_{1}$ and $m_{2}$ denote the spin projections of both neutrons and the momenta $\vec{p}, \vec{P}_{f}$ and $\vec{p}_{v}$ are the relative Jacobi momentum of outgoing nucleons, their total three-momentum and the muon neutrino momentum, respectively.

The matrix element for the muon capture on the three-nucleon bound state leading to a muon neutrino, a nucleon and the deuteron in the final state is expressed as

$$
N_{n d}^{\lambda}\left(m_{n}, m_{d}, m_{3 N}\right)=\left\langle\phi_{n d} \vec{q}_{0} m_{n} m_{d}\left|(1+P) j_{w}^{\lambda}\right| \Psi_{i}\right\rangle+\left\langle\phi_{n d} \vec{q}_{0} m_{n} m_{d}|P| U^{\lambda}\right\rangle .
$$

Here $\left|\phi_{n d} \vec{q}_{0} m_{n} m_{d}\right\rangle$ is a product state of the deuteron wave function and a momentum eigenstate of the spectator nucleon with the relative momentum $\vec{q}_{0}$ and $m_{n}, m_{d}$ and $m_{3 N}$ being the suitable spin projections. The auxiliary state $\left|U^{\lambda}\right\rangle$ fulfills the Faddeev-like equation [13]

$$
\left|U^{\lambda}\right\rangle=t G_{0}(1+P) j_{w}^{\lambda}\left|\Psi_{i}\right\rangle+t G_{0} P\left|U^{\lambda}\right\rangle .
$$


In the case of three free nucleons in the final state the nuclear matrix element $N^{\lambda}$ is also given by the auxiliary state $\left|U^{\lambda}\right\rangle$ :

$$
N^{\lambda}=\left\langle\Phi_{3 N}\left|(1+P) j_{w}^{\lambda}\right| \Psi_{i}\right\rangle+\left\langle\Phi_{3 N}|(1+P)| U^{\lambda}\right\rangle,
$$

where $\left|\Phi_{3 N}\right\rangle$ is an antisymmetrized state describing the free motion of the three outgoing nucleons.

Finally, in the case of the ${ }^{3} \mathrm{He}$ to ${ }^{3} \mathrm{H}$ transition

$$
N^{\lambda}\left(m_{3 H}, m_{3 H e}\right) \equiv\left\langle\Psi_{3 H} \vec{P}_{f}=-\vec{p}_{v} m_{3 H}\left|j_{w}^{\lambda}\right| \Psi_{3 H e} \vec{P}_{i}=0 m_{3 H e}\right\rangle,
$$

where initial ${ }^{3} \mathrm{He}$ is at rest $\left(\vec{P}_{i}=0\right.$ in the lab. system) and its spin projection is $m_{3 H e}$. The final ${ }^{3} \mathrm{H}$ nucleus moves with a momentum $\vec{P}_{f}$ opposite to the neutrino momentum $\vec{p}_{v}$ and its spin projection is $m_{3 H}$.

For the weak current $j_{w}^{\lambda}$ we employ a non-relativistic (NR) single nucleon current operator and supplement it by the $(p / M)^{2}$ relativistic corrections (RC), where $M$ is the nucleon mass. Denoting by $\vec{p}^{\prime}$ and $\vec{p}$ the nucleon momentum in the final and initial state, respectively, the weak current matrix elements are

$$
\begin{aligned}
\left\langle\vec{p}^{\prime}\left|j_{\mathrm{NR}+\mathrm{RC}}^{0}(1)\right| \vec{p}\right\rangle & =\left(g_{1}^{V}-\left(g_{1}^{V}-4 M g_{2}^{V}\right) \frac{\left(\vec{p}^{\prime}-\vec{p}\right)^{2}}{8 M^{2}}+\left(g_{1}^{V}-4 M g_{2}^{V}\right) \mathrm{i} \frac{\left(\vec{p}^{\prime} \times \vec{p}\right) \cdot \vec{\sigma}}{4 M^{2}}\right. \\
& \left.+g_{1}^{A} \frac{\vec{\sigma} \cdot\left(\vec{p}+\vec{p}^{\prime}\right)}{2 M}+g_{2}^{A} \frac{\left(\vec{p}^{\prime 2}-\vec{p}^{2}\right)}{4 M^{2}} \vec{\sigma} \cdot\left(\vec{p}^{\prime}-\vec{p}\right)\right) \tau_{-}
\end{aligned}
$$

and

$$
\begin{aligned}
\left\langle\vec{p}^{\prime}\left|\vec{j}_{\mathrm{NR}+\mathrm{RC}}(1)\right| \vec{p}\right\rangle & =\left(g_{1}^{V} \frac{\vec{p}+\vec{p}^{\prime}}{2 M}-\frac{1}{2 M}\left(g_{1}^{V}-2 M g_{2}^{V}\right) \mathrm{i} \vec{\sigma} \times\left(\vec{p}-\vec{p}^{\prime}\right)+g_{1}^{A}\left(1-\frac{\left(\vec{p}+\vec{p}^{\prime}\right)^{2}}{8 M^{2}}\right) \vec{\sigma}\right. \\
& \left.+\frac{g_{1}^{A}}{4 M^{2}}\left((\vec{p} \cdot \vec{\sigma}) \vec{p}^{\prime}+\left(\vec{p}^{\prime} \cdot \vec{\sigma}\right) \vec{p}+\mathrm{i}\left(\vec{p} \times \vec{p}^{\prime}\right)\right)+g_{2}^{A}\left(\vec{p}-\vec{p}^{\prime}\right) \frac{\vec{\sigma} \cdot\left(\vec{p}-\vec{p}^{\prime}\right)}{2 M}\right) \tau_{-}
\end{aligned}
$$

where we use the standard notation for spin $\sigma$ and isospin $\tau$ operators and $g_{1}^{V}, g_{1}^{A}, g_{2}^{V}, g_{2}^{A}$ are the nucleon weak form factors.

A more detailed discussion of the reaction kinematics, our model of weak current and its comparison with other approaches as well as formulas connecting the nuclear matrix elements with the capture rates are given in [16].

We solve Eqs. (2.5)- (2.8) using the formalism of partial waves. We employ all partial waves in the two-body systems up to the two-body total angular momentum $\mathrm{j}=3$ and in the three-body states up to the three-body total angular momentum $J=\frac{15}{2}$, see $[2,13]$ for the discussion of more technical details including convergence of our predictions.

The chiral approach gives us a unique opportunity to estimate one of the contributions to the uncertainty of theoretical predictions. In Ref. [21] the prescription for the estimation of the truncation errors, i.e. uncertainties arising from neglecting, at a given order of the chiral expansion, the contributions from higher orders, is given. We apply it also here and estimate the truncation 
error $\delta(X)^{(i)}$ of an observable $X$ at the $i$-th order of the chiral expansion, with $i=0,2,3, \ldots$ If $Q$ denotes the chiral expansion parameter, the expressions for truncation errors are

$$
\begin{aligned}
& \delta(X)^{(0)} \geq \max \left(Q^{2}\left|X^{(0)}\right|,\left|X^{(i \geq 0)}-X^{(j \geq 0)}\right|\right), \\
& \delta(X)^{(2)}=\max \left(Q^{3}\left|X^{(0)}\right|, Q\left|\Delta X^{(2)}\right|,\left|X^{(i \geq 2)}-X^{(j \geq 2)}\right|\right), \\
& \delta(X)^{(i)}=\max \left(Q^{i+1}\left|X^{(0)}\right|, Q^{i-1}\left|\Delta X^{(2)}\right|, Q^{i-2}\left|\Delta X^{(3)}\right|\right) \text { for } i \geq 3 .
\end{aligned}
$$

In the above formulas $X^{(i)}$ is a prediction for the observable $X$ at $i$-th order, $\Delta X^{(2)} \equiv X^{(2)}-X^{(0)}$ and $\Delta X^{(i)} \equiv X^{(i)}-X^{(i-1)}$ for $i \geq 3$. In addition we also require that $\delta(X)^{(2)} \geq Q \delta(X)^{(0)}$ and $\delta(X)^{(i)} \geq Q \delta(X)^{(i-1)}$ for $i \geq 3$.

\section{Results}

In Tabs. 1-4 we present results for the capture rates on ${ }^{2} \mathrm{H}$ and ${ }^{3} \mathrm{He}$ obtained with the chiral potential $[19,20]$ with the semi-local regularization for all orders of chiral expansion up to $\mathrm{N}^{4} \mathrm{LO}$ and for different values of the regulator $R$. In addition, in Tab. 5 we give the capture rates for the same processes but obtained with the AV18 nucleon-nucleon force [23] alone or supplemented by the Urbana IX [24] three-nucleon force. In Tab. 5 we also include results for the muon capture on ${ }^{3} \mathrm{H}$ with three free neutrons and a muon neutrino in the final state.

Comparing numbers from Tab. 5 with the corresponding results from Tabs. 1-4 (i.e. results with the AV18 potential only with the predictions at $\mathrm{N}^{4} \mathrm{LO}$ at $R=0.9 \mathrm{fm}$ ) we observe good overall agreement. Of course, due to the different dynamical models, one should not expect exactly the same numbers and indeed the observed differences are small. Results obtained with the semiphenomenological forces can also deliver an intuition about a role played by the three-nucleon force and meson exchange currents. For example, effects of the three-nucleon Urbana IX force are about $2 \%$ for the $\mu^{-}+{ }^{3} \mathrm{He} \rightarrow{ }^{3} \mathrm{H}+v_{\mu}$ capture. For the same process the dominant meson exchange currents changes the capture rate by about $5 \%$.

The capture rates arising from the chiral approach behave in a similar way. The convergence of predictions at a given regulator value is perfect in all cases. The differences between predictions at subsequent orders decrease and the changes between predictions at $\mathrm{N}^{4} \mathrm{LO}$ and $\mathrm{N}^{3} \mathrm{LO}$ are less then approximately $1 \%$ for all the processes. Of course this result is not a final proof of convergence of the complete chiral approach comprising also the chiral three-nucleon force (which contribute from $\mathrm{N}^{2} \mathrm{LO}$ ) and consistent, at given order, one- and many-body axial currents. However, since the two-body interaction and single nucleon current gives dominant contributions to capture rates our results give hope for a good convergence of predictions also in complete calculations.

To facilitate study of the cut-off dependence of chiral predictions in Tabs. 1-4 we include also the spread of the results at a given chiral order due to the different $R$ values, $\Delta$. Starting from the NLO, for all processes, $\Delta$ 's are very small compared to the absolute values of the capture rates. Moreover, removing results with $R=0.8 \mathrm{fm}$ and $R=1.2 \mathrm{fm}$, which yield the poorest description of the nucleon-nucleon phase shifts, leads to a further reduction of $\Delta$.

Finally, in Tabs. 1-4 we also show estimations for the truncation errors $\delta(\Gamma)$, obtained from Eqs. (2.11) for predictions with $R=0.9 \mathrm{fm}$. It is clear, that the expected contributions from higher orders of chiral expansion decrease when moving from a given order to the subsequent one. However, 


\begin{tabular}{|c|c|c|c|c|c|c|c|}
\hline chiral order & $R=0.8 \mathrm{fm}$ & $R=0.9 \mathrm{fm}$ & $R=1.0 \mathrm{fm}$ & $R=1.1 \mathrm{fm}$ & $R=1.2 \mathrm{fm}$ & $\delta(\Gamma)$ & $\Delta$ \\
\hline $\mathrm{LO}$ & 396.0 & 397.4 & 398.4 & 398.9 & 399.2 & 21.02 & 3.2 \\
$\mathrm{NLO}$ & 384.2 & 385.8 & 387.2 & 388.6 & 389.8 & 4.84 & 5.6 \\
$\mathrm{~N}^{2} \mathrm{LO}$ & 385.0 & 386.1 & 387.2 & 388.3 & 389.3 & 1.11 & 4.3 \\
$\mathrm{~N}^{3} \mathrm{LO}$ & 386.8 & 386.4 & 385.2 & 384.3 & 383.2 & 0.26 & 3.6 \\
$\mathrm{~N}^{4} \mathrm{LO}$ & 385.5 & 386.1 & 386.3 & 385.6 & 384.6 & 0.06 & 1.7 \\
\hline
\end{tabular}

Table 1: The capture rates $\Gamma$ in $\left[\mathrm{s}^{-1}\right]$ for the $\mu^{-}+\mathrm{d} \rightarrow \mathrm{n}+\mathrm{n}+v_{\mu}$ process obtained with the chiral nucleonnucleon interaction at given order and the $\mathrm{NR}+\mathrm{RC}$ model of the nuclear weak current operator. In the next-to-last column the truncation error $\delta(\Gamma)$ at given order of the chiral expansion for the capture rate, obtained for $R=0.9 \mathrm{fm}$, is given. In the last column the spread of the results at a given chiral order due to the different $R$ values, $\Delta$ in $\left[\mathrm{s}^{-1}\right]$, is shown.

\begin{tabular}{|c|c|c|c|c|c|c|c|}
\hline chiral order & $R=0.8 \mathrm{fm}$ & $R=0.9 \mathrm{fm}$ & $R=1.0 \mathrm{fm}$ & $R=1.1 \mathrm{fm}$ & $R=1.2 \mathrm{fm}$ & $\delta(\Gamma)$ & $\Delta$ \\
\hline $\mathrm{LO}$ & 1610 & 1618 & 1610 & 1594 & 1572 & 314.0 & 46 \\
$\mathrm{NLO}$ & 1330 & 1357 & 1381 & 1405 & 1427 & 72.2 & 97 \\
$\mathrm{~N}^{2} \mathrm{LO}$ & 1337 & 1356 & 1376 & 1395 & 1415 & 16.6 & 78 \\
$\mathrm{~N}^{3} \mathrm{LO}$ & 1314 & 1304 & 1289 & 1278 & 1266 & 3.8 & 48 \\
$\mathrm{~N}^{4} \mathrm{LO}$ & 1296 & 1307 & 1308 & 1299 & 1285 & 0.9 & 23 \\
\hline
\end{tabular}

Table 2: The same as in the Tab. 1 but for the $\mu^{-}+{ }^{3} \mathrm{He} \rightarrow{ }^{3} \mathrm{H}+v_{\mu}$ process.

\begin{tabular}{|c|c|c|c|c|c|c|c|}
\hline chiral order & $R=0.8 \mathrm{fm}$ & $R=0.9 \mathrm{fm}$ & $R=1.0 \mathrm{fm}$ & $R=1.1 \mathrm{fm}$ & $R=1.2 \mathrm{fm}$ & $\delta(\Gamma)$ & $\Delta$ \\
\hline $\mathrm{LO}$ & 262 & 282 & 312 & 350 & 392 & 304.0 & 130 \\
$\mathrm{NLO}$ & 536 & 525 & 515 & 504 & 492 & 69.9 & 44 \\
$\mathrm{~N}^{2} \mathrm{LO}$ & 547 & 539 & 529 & 518 & 507 & 16.1 & 40 \\
$\mathrm{~N}^{3} \mathrm{LO}$ & 584 & 586 & 592 & 596 & 603 & 3.7 & 19 \\
$\mathrm{~N}^{4} \mathrm{LO}$ & 590 & 584 & 583 & 587 & 595 & 0.9 & 12 \\
\hline
\end{tabular}

Table 3: The same as in the Tab. 1 but for the $\mu^{-}+{ }^{3} \mathrm{He} \rightarrow \mathrm{d}+\mathrm{n}+v_{\mu}$ process.

these numbers have to be considered with caution - in some cases the actual difference between predictions at two subsequent orders exceeds the estimated magnitude of truncation errors. It would be interesting to check how these numbers change after using a consistent (at each order) weak current operator.

Summarizing, we investigate the muon capture on the deuteron and the three-nucleon bound states focusing on applications of the chiral two-body force with the semi-local regularization. In the first applications of this force to the muon capture processes, presented here, we neglect the three-body interaction and restrict ourselves to only the single nucleon current. The obtained results, even under these simplifying assumptions, are very promising. The magnitudes of the capture rates are in good agreement with the predictions obtained with the AV18 two-nucleon force, the convergence of predictions with respect to the orders of chiral expansion is fast and the dependence of the capture rates on a magnitude of the regulator is extremely small. This provides a good background for future studies of the muon capture processes with a complete chiral Hamiltonian. 


\begin{tabular}{|c|c|c|c|c|c|c|c|}
\hline chiral order & $R=0.8 \mathrm{fm}$ & $R=0.9 \mathrm{fm}$ & $R=1.0 \mathrm{fm}$ & $R=1.1 \mathrm{fm}$ & $R=1.2 \mathrm{fm}$ & $\delta(\Gamma)$ & $\Delta$ \\
\hline $\mathrm{LO}$ & 95 & 99 & 105 & 113 & 120 & 70.0 & 26 \\
$\mathrm{NLO}$ & 159 & 157 & 154 & 151 & 148 & 16.1 & 11 \\
$\mathrm{~N}^{2} \mathrm{LO}$ & 161 & 159 & 157 & 154 & 151 & 3.7 & 10 \\
$\mathrm{~N}^{3} \mathrm{LO}$ & 169 & 169 & 171 & 172 & 175 & 0.9 & 6 \\
$\mathrm{~N}^{4} \mathrm{LO}$ & 170 & 169 & 169 & 170 & 173 & 0.2 & 4 \\
\hline
\end{tabular}

Table 4: The same as in the Tab. 1 but for the $\mu^{-}+{ }^{3} \mathrm{He} \rightarrow \mathrm{p}+\mathrm{n}+\mathrm{n}+v_{\mu}$ process.

\begin{tabular}{|c|c|c|}
\hline process & dynamical model & $\Gamma$ \\
\hline$\mu^{-}+\mathrm{d} \rightarrow \mathrm{n}+\mathrm{n}+v_{\mu}$ & AV18, with RC & 404 \\
\hline & AV18, with RC & 1295 \\
$\mu^{-}+{ }^{3} \mathrm{He} \rightarrow{ }^{3} \mathrm{H}+v_{\mu}$ & AV18+ Urbana IX, with RC & 1324 \\
& AV18+ Urbana IX with MEC [10] & 1386 \\
\hline$\mu^{-}+{ }^{3} \mathrm{He} \rightarrow \mathrm{d}+\mathrm{n}+v_{\mu}$ & AV18, with RC & 604 \\
& AV18+ Urbana IX & 544 \\
\hline$\mu^{-}+{ }^{3} \mathrm{He} \rightarrow \mathrm{p}+\mathrm{n}+\mathrm{n}+v_{\mu}$ & AV18, with RC & 169 \\
& AV18+ Urbana IX, with RC & 154 \\
\hline & AV18 & 37.5 \\
$\mu^{-}+{ }^{3} \mathrm{H} \rightarrow \mathrm{n}+\mathrm{n}+\mathrm{n}+v_{\mu}$ & AV18, with RC & 36.5 \\
& AV18+ Urbana IX, with RC & 32.6 \\
\hline
\end{tabular}

Table 5: The capture rates $\Gamma$ in $\left[\mathrm{s}^{-1}\right]$ for a muon capture on ${ }^{2} \mathrm{H},{ }^{3} \mathrm{H}$ and ${ }^{3} \mathrm{He}$ obtained with the AV18 nucleon-nucleon force alone or supplemented with the Urbana IX three-nucleon force.

\section{References}

[1] L.D.Faddeev, Sov. Phys. JETP 12 (1961) 1014 (Zh.Eksp.Teor.Fiz. 39, 1459 (1960).

[2] W. Glöckle, H.Witała, D. Hüber, H. Kamada, and J. Golak, Phys. Rept. 274, 107 (1996).

[3] H.Witała, T.Cornelius, W.Glöckle, Few-Body Syst. 3, 123 (1988).

[4] D.Hüber, H.Witała, W.Glöckle, Few-Body Syst. 14, 171 (1993).

[5] H.Witała, W.Glöckle, D.Hüber, J.Golak, H.Kamada, Phys. Rev. Lett. 81, 1183 (1998).

[6] H.Witała et al., Phys. Rev. C63, 024007 (2001).

[7] J.Kuroś-Żołnierczuk et al., Phys. Rev. C66, 024003 (2002).

[8] J.Carlson, R.Schiavilla, Rev. Mod. Phys. 70, 743 (1998).

[9] L. E.Marcucci et al., Phys. Rev. C83, 014002 (2011) and references therein.

[10] L. E.Marcucci, R. Schiavilla,M. Viviani, A. Kievsky, S. Rosati, and J. F. Beacom, Phys. Rev. C63, 015801 (2000).

[11] H.Krebs, E. Epelbaum, and Ulf-G. Meißner, arXiv:1610.03569 [nucl-th].

[12] H. Witała, J. Golak, R. Skibiński, and K. Topolnicki, J. Phys. G: Nucl. Part. Phys. 41, 094011 (2014).

[13] J. Golak, R. Skibiński, H. Witała, W. Glöckle, A. Nogga, and H. Kamada, Phys. Rept. 415, 89 (2005). 
[14] R.Skibiński, J.Golak, H.Witała, W.Glöckle, Phys. Rev. C59, 2384 (1999).

[15] J.Golak et al., Phys. Rev. C55, 2196 (1997).

[16] J.Golak et al., Phys. Rev. C90, 024001 (2014).

[17] J.Golak et al., Phys. Rev. C94, 034002 (2016).

[18] R.Skibiński et al., Phys. Rev. C93, 064002 (2016).

[19] E. Epelbaum, H. Krebs, and Ulf-G. Meißner, Eur. Phys. J. A51, 53 (2015).

[20] E. Epelbaum, H. Krebs, and Ulf-G. Meißner, Phys. Rev. Lett. 115, 122301 (2015).

[21] S. Binder et al., Phys. Rev. C93, 044002 (2016).

[22] A. Nogga, D. Hüber, H. Kamada, and W. Glöckle, Phys. Lett. B409, 19 (1997).

[23] R.B.Wiringa, V.G.J.Stoks, and R.Schiavilla, Phys. Rev. C51, 38 (1995).

[24] B.S.Pudliner et al., Phys. Rev. C56, 1720 (1997). 